\title{
Einführung in den geistes-, sozial- und kulturwissenschaftlichen Wissenstransfer
}

\author{
Markus Hayden \& Daniel Wutti
}

Universitäten sind als staatliche Bildungs- und Forschungseinrichtungen der Lehre und der Wissenschaft verpflichtet (Trencher et al. 2014). Entsprechend wird schon im ersten Paragraphen des österreichischen Universitätsgesetzes 2002 (UG 2002) explizit dargelegt, dass Universitäten dazu berufen sind, „der wissenschaftlichen Forschung und Lehre, der Entwicklung und der Erschließung der Künste sowie der Lehre der Kunst zu dienen“. Der darauffolgende Halbsatz präzisiert, dass der gesellschaftliche Auftrag der Universitäten damit jedoch noch nicht endet. So sind die Universitäten nämlich auch dazu verpflichtet, „zur Lösung der Probleme des Menschen sowie zur gedeihlichen Entwicklung der Gesellschaft und der natürlichen Umwelt beizutragen". Zudem wird festgehalten, dass auch die Förderung des wissenschaftlichen Nachwuchses mit dem Ziel einhergeht, „zur Bewältigung der gesellschaftlichen Herausforderungen in einer sich wandelnden humanen und geschlechtergerechten Gesellschaft beizutragen“ $(\$ 1)$.

Weitere, durch das UG 2002 gesetzlich definierte, Aufgaben der Universitäten inkludieren die „Unterstützung der Nutzung und Umsetzung ihrer Forschungsergebnisse in der Praxis und Unterstützung der gesellschaftlichen Einbindung von Ergebnissen der Entwicklung und Erschließung der Künste“ (\$3 Abs. 8) sowie die „Information der Öffentlichkeit über die Erfüllung der Aufgaben der Universitäten“ ( $\$ 3$ Abs. 11). Diese Pflichten gehören zur sog. universitären ,third mission 
und beinhalten unter anderem den Transfer von wissenschaftlichen Erkenntnissen zu außeruniversitären Feldern, wie Politik, Wirtschaft, Praxis und Zivilgesellschaft (Trencher et al. 2014; Zawdie 2010). In der EU-Strategie „Europa 2020“ sowie der Strategie der Bundesregierung für Forschung, Technologie und Innovation ${ }^{1}$ finden Begriffe wie ,Wissens- und Technologietransfer' zentrale Erwähnung. Sie stehen für die Suche nach Anwendungsfeldern für wissenschaftliche Erkenntnisse, für die Einbindung dieser Erkenntnisse in einen gesellschaftlichen Kontext sowie für die Sichtbarmachung des Nutzens der Wissenschaft für die Allgemeinheit.

Die wissenschaftliche Bearbeitung des Themenbereichs, Wissenstransfer' wird aktuell von Forschung hinsichtlich der Wissensvermittlung zu Wirtschaft und Industrie dominiert (siehe beispielsweise Geuna and Muscio 2009; Agrawal 2001; European Commission 2013 für Überblicksarbeiten). Andere Teilbereiche, wie etwa die Dissemination von Wissen zu Praxisfeldern und anderen gesellschaftlichen Sphären, wurden hingegen bisher nur unzureichend beforscht (Olmos-Peñuela, Benneworth, and Castro-Martinez 2014; Wutti and Hayden 2017). Während eine große Anzahl an Studien zu wissenschaftlichen Leistungen innerhalb der Scientific Community (Science to Science') sowie zum Wissens- und Technologietransfer im Sinne der Verwertung (Patentierungen, Lizensierungen, Spin-Offs, etc.) existiert, sind Forschungen über die Kommunikation bzw. Kooperation mit Praktiker_innen (,Science to Professionals') oder der breiteren Öffentlichkeit (Science to Public') sehr selten (Olmos-Peñuela, Castro-Martínez, and Manjarrés-Henríquez 2010; Hayden et al. 2018). In Anbetracht der Tatsache, dass Wirtschaftskooperationen sowie Themen der Verwertung nur einen marginalen Anteil des breiten Wissenstransferspektrums einnehmen (Hayden, Petrova, and Wutti 2018), erscheint die Schieflage in der Forschung in gewissem Maße

\footnotetext{
${ }^{1}$ Siehe: https://bit.ly/2QxuoR5 [Stand: 10.12.2018]
} 
abwegig. Vor allem in den Geistes-, Sozial- und Kulturwissenschaften (GSK-Wissenschaften) greift der Fokus auf wirtschaftliche Aspekte des Wissenstransfers deutlich zu kurz. Die GSK-Wissenschaften folgen anderen Forschungstraditionen als beispielsweise die sog. MINTFächer (Mathematik, Informatik, Naturwissenschaft und Technik). So sind etwa Themen sozialer Relevanz oder die Suche nach nachhaltigen Lösungen für globale Herausforderungen häufige Betätigungsfelder geistes-, sozial- und kulturwissenschaftlicher Forschung (Van Langenhove 2012). Zudem besteht oftmals ein stärkeres Interesse an Bewusstseinsbildung und gesellschaftlicher Weiterentwicklung (Wutti and Hayden 2017). Diese und weitere Faktoren erschweren die vergleichende Evaluierung des Wissenstransfers in den unterschiedlichen wissenschaftlichen Disziplinen (Olmos-Peñuela, Benneworth, and Castro-Martinez 2014).

Die Bedeutung von Wissenstransfer in den GSK-Wissenschaften soll an dieser Stelle exemplarisch anhand der Suche nach Lösungen globaler Problemstellungen aufgezeigt werden. Die GSK-Wissenschaften leisten in diesem Bereich einen besonderen Beitrag, da sie gesellschaftliche Fragestellungen und Herausforderungen in ihrer Relevanz und Aktualität beforschen. Sie erarbeiten nachhaltige Handlungsoptionen und sozial verträgliche strategische Maßnahmen, welche für ein weiteres Vorgehen unerlässlich sind (Van Langenhove 2012). Zur praktischen Umsetzung der Vorschläge bedarf es jedoch mehr als Forschung alleine. Ohne eine adäquate Dissemination der Ergebnisse, wie etwa durch Beratung von Politiker_innen und anderen Entscheidungsträger_innen bzw. Gremien und Ausschüssen, besteht das Risiko, dass die Forschungsergebnisse unbeachtet in wissenschaftlichen Bibliotheken verstauben. Auch die nötige Aufklärung der Gesamtbevölkerung hinsichtlich obligatorischer Änderungen des Lebenswandels, Themen der sozialen Gerechtigkeit oder verantwortungsvollem Umgang mit natürlichen Ressourcen stellen verbindliche Elemente notwendiger Bewusstseinsbildung dar (Jasanoff 2012; Vucetich and Nelson 2010). 
All diese Bereiche sind praktische Anwendungsfelder von GSKWissenstransfer.

Unsere bisherigen Forschungen im Rahmen des Wissenstransferzentrums Süd zeigen, dass Wissenschafter_innen der GSK-Disziplinen beachtliche Wissenstransferleistungen vollbringen, diese aber im universitären Geschäft nur selten Beachtung erfahren (Hayden et al. 2018). Die Leistungen sind kaum sichtbar und erhalten in der Scientific Community nur geringe Wertschätzung. Dies ist unter anderem dadurch begründet, dass Wissenstransferleistungen nicht adäquat in Forschungsdokumentationen erfasst oder durch Kennzahlen abgebildet werden (siehe hierzu auch Abschnitt 7.1 in diesem Issue). Während es genaue Kennziffern und Benchmarks für Leistungen im Bereich ,Science to Science' gibt, wurden die Bereiche ,Science to Professionals' und ,Science to Public' lange Zeit nicht beachtet. Erst seit wenigen Jahren gibt es Bestrebungen, auch Errungenschaften aus dem Bereich Wissenstransfer an den österreichischen Universitäten zu erfassen. So existieren inzwischen Kennzahlen für die Anzahl der Publikationen und Vorträge, die für Praktiker_innen oder Laien konzipiert wurden (Wutti and Hayden 2017).

In diesem Special Issue haben wir einige Texte zusammengefasst, die einen Einblick in das umfangreiche Themenfeld des Wissenstransfers in den Geistes-, Sozial- und Kulturwissenschaften gewähren. Dazu stellen wir neue Forschungsergebnisse und konkrete Aufgabenbereiche, wie die Themenbereiche, Open Access ‘ oder ,Gender \& Empowerment', vor. Zudem werden Hindernisse und Förderungsmöglichkeiten thematisiert. Neben Good-Practice-Beispielen inkludiert die Publikation auch Erfahrungsberichte von anderen Wissenstransferzentren. Die Texte sollen für das Thema sensibilisieren und verdeutlichen, dass Wissenstransfer einen essenziellen Bestandteil des 
universitären Alltages darstellt. Des Weiteren sollen sie durch eine differenziertere Betrachtungsweise zu einem besseren Verständnis der Thematik beitragen. 


\section{Literatur}

Agrawal, Ajay 2001. "University-to-Industry Knowledge Transfer:

Literature Review and Unanswered Questions." International

Journal of Management Reviews 3 (4): 285-302.

doi:10.1111/1468-2370.00069.

European Commission. 2013. Knowledge Transfer Study 2010-2012.

Brussels: European Union. doi:10.2777/31336.

Geuna, Aldo, and Alessandro Muscio. 2009. "The Governance of University Knowledge Transfer: A Critical Review of the Literature." Minerva 47 (1): 93-114. doi:10.1007/s11024009-9118-2.

Hayden, Markus C, Margarita K Petrova, and Daniel Wutti. 2018.

"Direct Associations of the Terminology of Knowledge

Transfer - Differences Between the Social Sciences and

Humanities (SSH) and Other Scientific Disciplines." Trames:

A Journal of the Humanities and Social Sciences 22 (3): 239-56. doi:10.3176/tr.2018.3.02.

Hayden, Markus C, Martin Weiß, Alice Pechriggl, and Daniel

Wutti. 2018. "Insights Into University Knowledge Transfer in the Social Sciences and Humanities (SSH) and Other

Scientific Disciplines - More Similarities Than Differences.”

Frontiers in Research Metrics and Analytics 3: 32.

doi:10.3389/frma.2018.00032.

Jasanoff, Sheila. 2012. Science and Public Reason. New York, NY:

Routledge.

Langenhove, Luk Van. 2012. "Global Issues: Make Social Sciences

Relevant.” Nature 484 (7395): 442-442.

doi:10.1038/484442a. 
Olmos-Peñuela, Julia, Paul Benneworth, and Elena Castro-Martinez. 2014. “Are 'STEM from Mars and SSH from Venus'?:

Challenging Disciplinary Stereotypes of Research's Social Value." Science and Public Policy 41 (3): 384-400. doi: $10.1093 /$ scipol/sct071.

Olmos-Peñuela, Julia, Elena Castro-Martínez, and Liney Adriana Manjarrés-Henríquez. 2010. “Knowledge Transfer In Humanities And Social Science Research Groups: The Relevance Of Organizational Factors." Ingenio Working Paper Series 2010/15.

Trencher, Gregory, Masaru Yarime, Kes B. McCormick, Christopher N H Doll, and Steven B. Kraines. 2014. "Beyond the Third Mission: Exploring the Emerging University Function of Co-Creation for Sustainability." Science and Public Policy 41 (2): 151-79. doi:10.1093/scipol/sct044.

Vucetich, John A, and Michael P Nelson. 2010. “The Moral Obligations of Scientists." Minding Nature 3 (2): 48-50.

Wutti, Daniel, and Markus Hayden. 2017. "Knowledge Transfer in the Social Sciences and Humanities (SSH) - Definition, Motivators, Obstacles, and Visions." Colloquium: New Philologies 2 (1): 87-101. doi:10.23963/cnp.2017.2.1.7.

Zawdie, Girma. 2010. "Knowledge Exchange and the Third Mission of Universities.” Industry \& Higher Education 24 (3): 151-55. doi:10.5367/000000010791657437. 